Voix et Images

voixetimages

\title{
Les Aires de la chanson québécoise
}

\section{Robert Saint-Amour}

Volume 11, numéro 2 (32), hiver 1986

Michel van Schendel

URI : https://id.erudit.org/iderudit/200562ar

DOI : https://doi.org/10.7202/200562ar

Aller au sommaire du numéro

\section{Éditeur(s)}

Université du Québec à Montréal

\section{ISSN}

0318-9201 (imprimé)

1705-933X (numérique)

Découvrir la revue

\section{Citer cet article}

Saint-Amour, R. (1986). Les Aires de la chanson québécoise. Voix et Images, 11(2),

325-327. https://doi.org/10.7202/200562ar d'utilisation que vous pouvez consulter en ligne.

https://apropos.erudit.org/fr/usagers/politique-dutilisation/ 


\title{
Les Aires de la chanson québécoise
}

\author{
par Robert Saint-Amour, Université du Québec à Montréal
}

\section{Un aperçu général}

Les Aires de la chanson québécoise ${ }^{1}$ (en musique, homophonie oblige) est un ouvrage publié sous la direction de Robert Giroux avec la participation de: Jacques Julien, Renée-Berthe Drapeau, Bruno Roy et Jean-Jacques Schirra. Il est à la fois le résultat d'une longue réflexion individuelle des co-auteurs et le point de départ collectifi, une ébauche de recherche de longue haleine $^{2}$ qui considère la chanson comme un objet complexe et en aborde l'analyse comme tel. Car, ainsi que le souligne Robert Giroux, dans la chanson ce n'est pas le sens des mots qui est déterminant, mais bien la chanson dans toutes ses composantes ${ }^{3}$.

Une telle affirmation n'est pas négation de la valeur et de la fonction des mots dans la chanson. Au contraire, elle est manifestation d'une volonté de replacer le texte (les mots d'une chanson) dans un contexte élargi, de rappeler que la signification ne peut être totale que dans la mesure où il est aussi, et en même temps, un objet musical, technique, esthétique, social, politique et économique ${ }^{4}$.

En elle-même, cette conception de la chanson pourrait être banale aux yeux d'un lecteur moderne tellement cela semble être l'évidence même, tellement ces différents aspects font l'objet de discours spécialisés. Cependant, dans le domaine de la chanson québécoise, les choses n'ont pas toujours été aussi claires.

Nul doute que quelques-uns des jalons méthodologiques contenus dans les Aires... ont été soulevés dans des ouvrages ou des articles antérieurs. Mais ils l'étaient de façon informelle et, pour cette raison même, trop souvent inopérationnels, perdus qu'ils étaient dans une masse d'informations historiques, biographiques (ou anecdotiques), thématiques ou autres. Il y a encore à peine cinq ans, la recherche avançait à pas de tortue dans ce domaine parce que la chanson était un objet d'étude dévalorisé par la presque totalité des intellectuels québécois. Tout était à créer; tout l'est encore.

Voilà pourquoi une fois replacé dans cette perspective, l'effort théorique annoncé et amorcé par les Aires... est beaucoup plus important qu'il ne le paraît à première vue. Il pose comme principe de base que la compréhension du référent musical est inséparable de la situation d'énonciation et de récep- 
tion du groupe culturel dont il émane, et que, par conséquent, les effets de ce même référent sont d'autant plus signifiants que le groupe producteur est homogène ${ }^{6}$.

C'est donc dans cet esprit que tous les textes de cet ouvrage parleront du discours de la chanson elle-même ou du discours sur la chanson ou du degré de communication entre la vedette, sa performance et son public ${ }^{7}$. Si certaines de ces analyses paraissent incomplètes aux yeux et au gré d'un lecteur averti, on ne peut que le regretter. Elles n'en demeurent pas moins une tentative louable de définition d'un objet trop longtemps assimilé au texte littéraire sans être pour autant reconnu par cette même institution littéraire.

\section{Des textes eux-mêmes}

Responsable de l'ouvrage, donc de l'introduction, Robert Giroux est l'auteur de trois articles à travers lesquels on retrouve certains des principes méthodologiques autour desquels est axée la recherche.

Ainsi, la présentation de la programmation du Colloque international sur la chanson tenu à Sainte-Baume, en novembre 19838, devient-elle l'occasion d'un compte rendu commenté qui expose aussi bien les prises de position des intervenants ou leurs hypothèses de travail que celles de l'auteur lui-même. Dans "Le dépôt sonore. Trois fragments d'un texte à venir»", partant d'expériences et de réflexions personnelles très spontanées (peut-être sont-elles trop anecdotiques d'ailleurs?), Giroux est arrivé à établir la distinction entre sujet parlant et sujet musicant [sic] dans le but de montrer que la fonction musicale, tout en étant exercée par un petit groupe de compositeurs, conditionne profondément les goûts des sociétés. La chanson actuelle en est la preuve la plus évidente. Puis en quinze pages, «Les deux pôles de la chanson québécoise: de la chanson westerne à la chanson contre-culturelle ${ }^{10}$ rappelle la place importante occupée depuis des décennies par le phénomène western dans notre société québécoise, son caractère fondamentalement a-historique, a-politique et éternel comme la ruralité". Objet de dérision et de critique de la part des intellectuels et de la chanson contreculturelle à prétention révolutionnaire ${ }^{12}$, comment réagira-t-elle face au nouvel envahisseur qu'est le rock?

Sans pour autant négliger son «Essai de typologie de la chanson populaire ${ }^{13}$ qui clarifie des positions et circonscrit les territoires de la chanson populaire contemporaine ${ }^{14}$, Jean-Jacques Julien livre, par ailleurs, d'intéressantes et de fort originales réflexions dans "Le maniérisme vocal ou la voix porteuse ${ }^{15}$. S'inspirant de notions sur la voix empruntées à l'opéra, il montre que la voix, un des supports (trop longtemps négligé!) de l'encodage,base et responsable de la communication verbale dans la chanson, est productrice de sens, engendre un maniérisme verbal, c'est-à-dire un comportement linguistique constitué de mille et une petites choses par lesquelles s'opère la reconnaissance immédiate des implications sociales d'un chanteur. Selon Julien, ce sont elles qui fondent l'interaction constante entre le chanteur et son public, ce qu'il illustre dans et par son analyse de la performance vocale de Robert Charlebois. 
"Attitudes linguistiques des jeunes et chanson québécoise»16 discute du rapport langue/musique rock (ou populaire) anglo-américaine en le situant au cœur même de l'environnement culturel quotidien des jeunes Québécois. Article de choc qui ne craint pas: de contester l'interprétation du Conseil de la langue française à propos d'une enquête sur la langue menée auprès des jeunes; d'affirmer qu'à une langue ne correspond plus une seule et unique culture ${ }^{17}$; de souligner l'absence d'implication politique des jeunes; de parler de l'indifférence de ces mêmes jeunes à l'égard de la chanson québécoise telle qu'entendue traditionnellement, parce qu'ils la disent incapable de renouvellement. Autant de miroirs qui nous retournent brutalement l'image de notre société québécoise, de la dualité des sources et de l'influence étrangère [qui] demeure une constante ${ }^{18}$. Qu'est-ce que chanter en québécois? Sur plus d'un point, le lecteur risque de diverger d'opinion avec l'auteur.

L'article de Jean-Jacques Schirra, "Les éditions sonores au Québec [ 1898-1960 ]» "19, et celui de Renée-Berthe Drapeau, "Le yé-yé dans la marge du nationalisme québécois [1960-1974] ${ }^{20}$, deviennent des documents précieux pour la recherche. Chacun, à sa façon, jette un meilleur éclairage sur des secteurs négligés de la chanson québécoise.

Peu importe qu'on soit en parfait accord ou en total désaccord avec le contenu de cet ouvrage, avec les opinions émises par les auteurs. Ce qui compte, c'est qu'il suscite maintenant la discussion et la recherche.

1. Montréal, Éditions Tryptique, 1984, 213 p.

2. Ibid., p. 11.

3. Ibid., p. 56

4. Ibid., p. 11

5. Ibid., p. 57.

6. Ibid., p. 57.

7. Ibid., p. 11.

8. Ibid., p. 17.

9. Ibid., p. 41.

10. Ibid., p. 153.

11. Ibid., p. 157.

12. Ibid., p. 169.

13. Ibid., p. 103.

14. Ibid., p. 105.

15. Ibid., p. 125 .

16. Ibid., p. 59.

17. Ibid., p. 64.

18. Ibid., p. 75 .

19. Ibid., p. 79.

20. Ibid., p. 175. 